\title{
SISTEMÁTICA E DISTRIBUIÇĀO DO COMPLEXO \\ DE ESPÉCIES Cynolebias minimus (CYPRINODONTIFORMES, RIVULIDAE), COM A DESCRIÇĀO DE DUAS ESPÉCIES NOVAS
}

Wilson J. E. M. Costa ${ }^{1}$

\begin{abstract}
In this paper the Cynolebias minimus species-complex is defined, together with a redescription of this species and a description of two new species. Informations on their ecology and reproductive behavior is also included. The interrelationships and distribution of the group is discussed.
\end{abstract}

\section{INTRODUÇÃO}

O gênero Cynolebias Steindachner, 1876, compõe-se de peixes de ciclo de vida anual, caracterizados por possuirem canal pré-opercular fechado e nadadeira caudal sem escamas (Parenti, 1981). Myers (1952), descreveu o subgênero Leptolebias, caracterizado pela forma delgada do corpo, tamanho reduzido e padrão uniforme de colorido das fêmeas.

Corpo delgado é o estado plesiomórfico em rivulídeos e tamanho reduzićo ocorre em outras espécies de Cynolebias não incluidas em Leptolebias. Entretanto, padrão de colorido uniforme em fêmeas, sem pintas, máculas ou faixas é um estado de ocorrência única na família.

C. minimus Myers, 1942, difere das demais Leptolebias spp. pelos machos que apresentam nadadeiras caudais ligeiramente truncadas, ao contrário de ovalada ou lanceolada.

La Corte (1982), observou variações de coloração em populações de Cynolebias minimus, considerando-as todas como provável única espécie, por obter prole fértil nos cruzamentos realizados em laboratório.

Após o exame de populações de 6 localidades das baixadas do Estado do Rio de Janeiro, C. minimus e mais duas espécies novas foram reconhecidas.

No presente trabalho $C$. minimus é redescrita e as duas espécies novas são descritas, acrescentando-se considerações sobre ecologia, comportamento reprodutivo, filogenia e distribuição.

\section{MÉTODOS}

Contagens foram feitas da seguinte maneira:

- raios das nadadeiras dorsal e anal - número total de elementos observados;

1 Departamento de Biologia Marinha, UFRJ - Pós-graduação USP. 
Revta bras. Zool.

- escamas na linha longitudinal - número de escamas desde aquela sobre a cintura escapular e imediatamente acima da abertura opercular, até a última sobre a placa hipo-ural (detectada pelo dobramento da base da nadadeira caudal);

- escamas em torno do pedúnculo - número de escamas numa linha circundando o pedúnculo caudal.

As medições foram registradas utilizando-se um paquímetro de $0,05 \mathrm{~mm}$ de precisão e depois ajustadas para $0,1 \mathrm{~mm}$. O procędimento escolhido foi:

- comprimento padrão - distância entre a ponta do focinho (aqui se referindo ao meio do limite posterior da depressão entre a maxila superior e o neuro-crânio) e o final da placa hipo-ural;

- altura do corpo (alt. corpo) - medida na vertical que passa pela região entre o ânus e a papila urogenital;

- comprimento da cabeça (comp. cab.) - maior distância entre a ponta do focinho e a margem posterior do osso opercular;

- largura da cabeça (larg. cab.) - maior largura entre os opérculos;

- altura da cabeça (alt. cab.) - comprimento da vertical que passa pelo ponto mais posterior da borda do osso opercular;

- diâmetro do olho (diam. olho) - medido horizontalmente;

- comprimento pré-dorsal (comp. p.-dor.) - distância entre a ponta do focinho e a parte posterior da base do último raio da nadadeira pélvica;

- altura do pedúnculo (alt. ped.) - menor vertical passando pela região entre a base da nadadeira dorsal e a nadadeira caudal;

- comprimento da base da nadadeira dorsal (c.bas.n.dor.) - distância entre a parte anterior da base do primeiro e a parte posterior da base do último raio da nadadeira;

- comprimento da base da nadadeira anal (c.bas.n.an.) - da mesma maneira feita na dorsal.

Valores merísticos são apresentados segundo a distribuição de freqüência. Valores morfométricos são expressos em variações de porcentagens do comprimento padrão, com exceção do diâmetro do olho, em porcentagens do comprimento da cabeça.

As abreviaturas citadas no material examinado significam: CAGC - coleção particular do Prof. Carlos Alberto G. Cruz, depositada na Universidade Federal Rural do Rio de Janeiro; MNRJ - Museu Nacional do Rio de Janeiro; e, MZUSP - Museu de Zoologia da Universidade de São Paulo.

\section{Cynolebias minimus Myers, 1942}

Figs. 1 e 4a-c

Cynolebias minimus Myers, 1942:109-110 (desc. orig., taboal $1 \mathrm{mi}$ L Itaquahy [Itaguaí], RJ, Brasil); 1952:129-130, 139 (cit.); Fowler, 1954:215 (catál.); Carvalho, 1957:461 (cit.); Vaz-Ferreira et al., 1964:33 (cit.); Weitzman \& Wourms, 1967:92-94 (caract. morfol.); Vaz-Ferreira \& Sierra, 1973:253254 (ilust.); Cruz, 1974:21 (cit.); 1983:73 (cit.); La Corte, 1980:11 (cit.); 1982:215-220, 226 (cor, ecol., hibridiz. e ilust.); Remeur, 1981a:33-34 (cit.); 1982b:22 (cit.); Lazara, 1982:166-184 (cit.); 1984-57 (catál.); Cruz \& Peix oto, 1983:93 (cit.). 
Vol. 5(4), 1988

Cynolebias (Cynopoecilus) splendens (non Cynolebias spendens Myers, 1942);

Ladiges, 1955:230 (10 export.); Schroeter, 1956:63-66 (erros de ident.). Cynopoecilus splendens (non Cynolebias splendens Myers, 1942); Siegel, 1958: 200-202 (ilust. ovo) (erro de ident.).

Cynolebias ladigesi Foersch, 1958:257-260 (desc. orig., poças d’água, 80 km NO do Rio de Janeiro); Weitzman, 1962:12-13 (nomencl.); Vaz-Ferreira et al., 1964:33 (cit.); Weitzman \& Wourms, 1967:93-94 (caract. morfol.); VazFerreira \& Sierra, 1973:252 (cit.); 1974:13-14 (cit.); Parenti, 1981:491 (lista mat. exam.); Remeur, 1981a:33-34 (cit.); 1981b:26 (obs. cativ.); 1981c:24 (cit.); 1982a:23,25 (manut. cativ., ilustr.); 1982b:22-23 (cit.); La Corte, 1982:218 (hibridiz.).

Cynopoecilus minimus; Seegers, 1980:134 (sinon.).

Cynolebias opalescens (non Cynolebias opalescens Myers, 1942); Huber, 1981:

6-7 (erro sinon.).

Diagnose: espécie muito semelhante a $C$. fractifasciatus sp. n. em caracteres merísticos e morfométricos, diferindo desta e de $C$. cruzi pelos machos que apresentam faixas verticais escuras da nadadeira caudal mais largas (fig. 4), contínuas e bem delimitadas, contrastando fortemente com o fundo azul-claro-esverdeado e geralmente sendo curvas com a parte côncava situada posteriormente. Estas faixas verticais são de coloração castanha ligeiramente avermelhada, enquanto nas outras duas espécies, são vermelho-pardo-escuras. $C$. minimus difere também pelos machos adultos que raramente apresentam faixas escuras verticais no corpo (machos jovens sempre as possuem), enquanto nas outras espécies geralmente elas são mantidas.

Descrição dos machos: exemplares examinados entre 14,4 e $26,4 \mathrm{~mm}$ de comprimento padrão.

Flanco, região opercular e íris de coloração verde-claro-metálica, com cerca de 14 faixas verticais no tronco (geralmente apenas nos jovens), três na região opercular, uma atravessando o olho e contorno das escamas de coloração castanho-avermelhada. Desta cor, cerca de 6 faixas verticais na nadadeira caudal (quase sempre curvas, com a parte côncava situada posteriormente), nove na anal e nove na dorsal, contrastam com fundo azul-claro-esverdeado. Pélvicas avermelhadas e peitorais transparentes.

Nadadeiras dorsal e anal terminando em pontas, caudal algo truncada (fig. 4) e peitorais de contorno arredondado. Limite posterior das peitorais sobre a vertical que passa pela papila uro-genital. Pélvica quando dobrada para trás chegando entre o 10 e 30 raio da anal. Origem da dorsal na vertical do 4 o ou 5 o raio da anal. Peitoral com papilas pouco conspícuas na parte anterior da face interna dos raios superiores.

Dados merísticos são apresentados na tab. 1 (com exceção das escamas em torno do pedúnculo que são $12 \mathrm{em}$ todos os exemplares) e dados morfométricos na tab. 2.

Descrição das fêmeas: exemplares examinados entre 16,3 e $23,6 \mathrm{~mm}$ de comprimento padrão.

Coloração do corpo castanho-claro-uniforme, com nadadeiras transparentes. Região opercular e porção anterior ventral rosadas e com reflexos verde- 
claros. Iris amarela e uma faixa vertical castanho-clara atravessando o olho. Nadadeira dorsal terminando em ponta e anal arredondada. Caudal ovalada ou algo truncada e contorno da peitoral arredondado. Limite posterior da peitoral sobre a vertical que passa pela base da pélvica, e esta quando dobrada para trás alcançando a base da papila uro-genital. Origem da dorsal na vertical do 2 ? , 3 ? ou 4 ? raio da anal.

Dados merísticos são apresentados na tab. 1 (com exceção das escamas em torno do pedúnculo que são $12 \mathrm{em}$ todos os exemplares) e dados morfométricos na tab. 2.

Comportamento reprodutivo (em parte já descrito e ilustrado por Foersch (1958)): o macho faz a corte em movimentos rápidos, enrolando as abas posteriores das nadadeiras dorsal e anal e eriçando-as num plano perpendicular à coluna vertebral. Durante estes movimentos efetuados em torno de uma fêmea, a nadadeira caudal do macho mantém-se um pouco fechada e o corpo fica ligeiramente encurvado. Logo após, o macho imerge a parte anterior do corpo no substrato, enquanto as nadadeiras fazem rápidos e ritmados movimentos de retração. Nesse momento uma fêmea se coloca lateralmente junto ao macho, e este, com o corpo sinuosamente encurvado, comprime a fêmea contra o substrato com um de seus flancos, dando-se a postura.

Forma do ovo: como descrito e ilustrado por Siegel (1958), é redondo e com aproximadamente $1 \mathrm{~mm}$ de diâmetro, apresentando o córion com prolongamentos em forma de cogumelo e com o comprimento cerca de $22,5 \%$ do raio do ovo ( 5 ovos foram examinados).

Notas ecológicas: encontradas em brejos de águas temporárias, com profundidade máxima de $60 \mathrm{~cm}$, água escura (cor de chá), fundo de lama e vegetação predominantemente de tifáceas e gramíneas. Vive em simpatria com Callichthys callichthys L., 1758, sendo uma vez encontrado um exemplar macho de uma espécie similar a Cynolebias nanus Cruz \& Peixoto, 1984, pelo Sr. Marco Túlio C. Lacerda, no Horto Florestal Santa Cruz, na localidade de Seropédica, Município de Itaguaí.

Distribuição: brejos na região noroeste da cidade do Rio de Janeiro, compreendendo os municípios do Rio de Janeiro e Itaguaí (fig. 5).

Material examinado: Seropédica, Mun. Itaguaí, RJ - MZUSP 36296 (4 ơ,

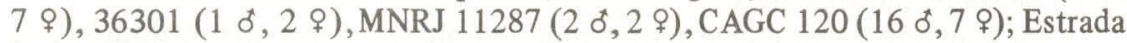
Pedra de Guaratiba - Santa Cruz, Mun. Rio de Janeiro, RJ - CAGC 119 (5 ơ, 5 \%), $122(10)$.

Material examinado mas não utilizado para contagens e medições: Capoeira Grande, Mun. Rio de Janeiro, RJ - CAGC 121 (3); Seropédica, Mun. Itaguaí, RJ - MNRJ 11286 (55), 11288 (10), CAGC 125 (15); Rodovia Presidente Dutra, km 37, Mun. Itaguaí, RJ - CAGC 118 (21).

FIGS. $1-3-1$, Cynolebias minimus Myers, 1942 (MNRJ 11287). Acima, macho com 23,0 mm de comprimento padrão e, abaixo, fêmea com 21,6mm. 2, Cynolebias fractifasciatus sp. n. (MNRJ 11292, parátipos). Acima, macho com 19,4mm de comprimento padrão e, abaixo, fêmea com $18,4 \mathrm{~mm}$. 3, Cynolebias cruzi sp. n. (MNRJ 11291, parátipos). Acima, macho com $22,1 \mathrm{~mm}$ de comprimento padrão e, abaixo, fêmea com $20,8 \mathrm{~mm}$. 


\section{Vol. 5(4), 1988}
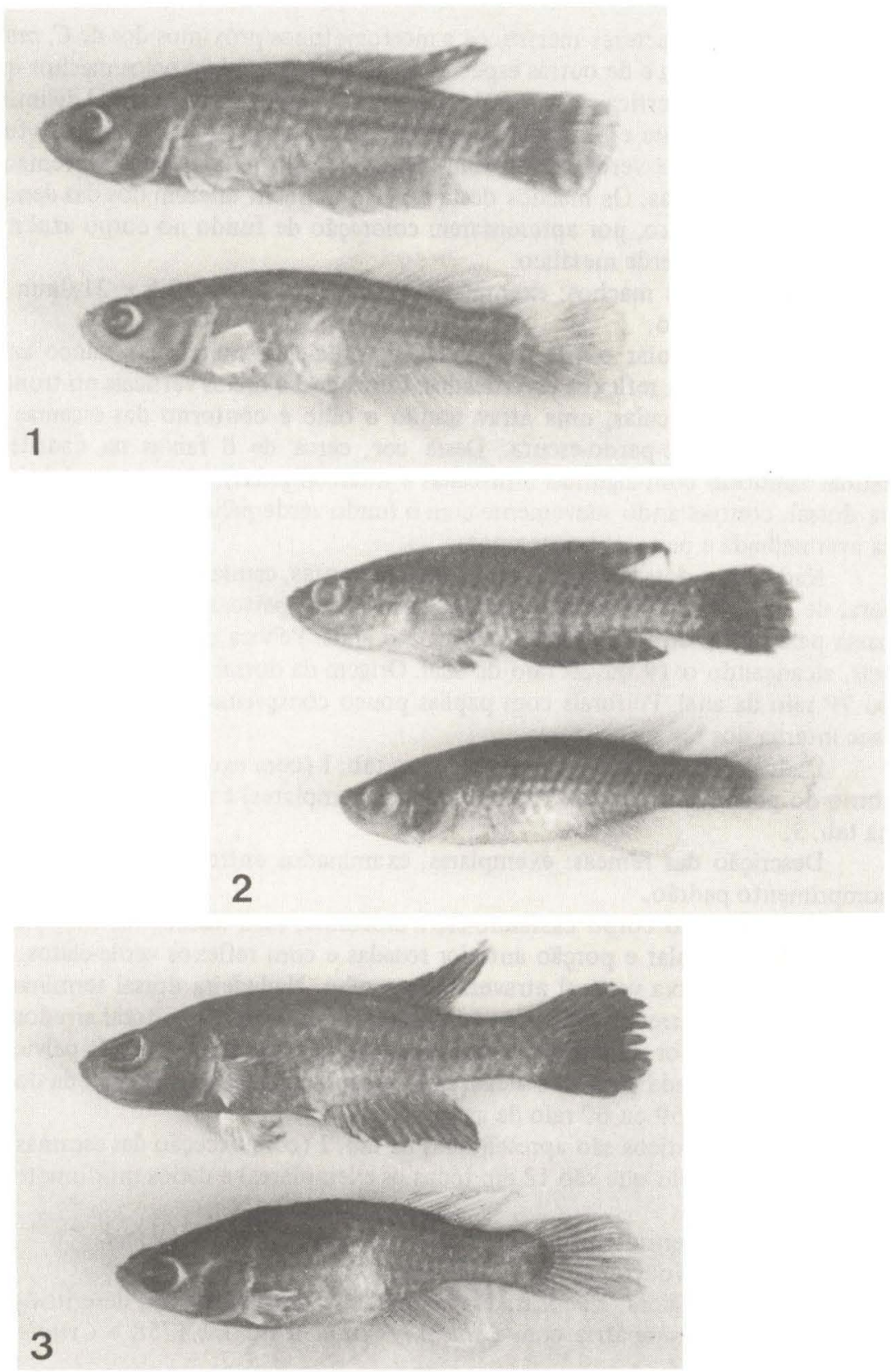

561 
Cynolebias fractifasciatus sp. $\mathbf{n}$.

Figs. 2 e 4d-f

Diagnose: Caracteres merísticos e morfométricos próximos dos de C. minimus, diferindo desta e de outras espécies do grupo Leptolebias pelos machos que apresentam faixas verticais escuras da nadadeira caudal tortuosas e mal delimitadas, com interrupções e bifurcações (fig. 4). Ao contrário de C. minimus, o fundo desta nadadeira é verde pálido translúcido, contrastando apenas suavemente com as faixas escuras. Os machos desta espécie também diferem dos das demais espécies do complexo, por apresentarem coloração de fundo no corpo azul metálico, ao invés de verde metálico.

Descrição dos machos: exemplares examinados entre 13,6 e $21,0 \mathrm{~mm}$ de comprimento padrão.

Região opercular e íris de coloração verde-claro-metálica e flanco azulclaro-metálico, com reflexos esverdeados. Cerca de 14 faixas verticais no tronco, três na região opercular, uma atravessando o olho e contorno das escamas de coloração vermelho-pardo-escura. Desta cor, cerca de 6 faixas na nadadeira caudal (sinuosas com algumas bifurcadas e interrompidas), nove na anal e nove na dorsal, contrastando suavemente com o fundo verde pálido translúcido. Pélvica avermelhada e peitoral transparente.

Nadadeira dorsal e anal terminando em pontas, caudal algo truncada e peitoral de contorno arredondado. Limite posterior da peitoral sobre a vertical que passa pela região entre a base da pélvica e o ânus. Pélvica quando dobrada para trás, alcançando o 1 ? ou 2 ? raio da anal. Origem da dorsal na vertical do 5 ?, 6 ? ou 7.0 raio da anal. Peitorais com papilas pouco conspícuas na parte interior da face interna dos raios superiores.

Dados merísticos são apresentados na tab. 1 (com exceção das escamas em torno do pedúnculo que são $12 \mathrm{em}$ todos os exemplares) e dados morfométricos na tab. 3 .

Descrição das fêmeas: exemplares, examinados entre 14,2 e $18,4 \mathrm{~mm}$ de comprimento padrão.

Coloração do corpo castanho-claro-uniforme, com nadadeiras transparentes. Região opercular e porção anterior rosadas e com reflexos verde-claros. Íris amarela e uma faixa vertical atravessando o olho. Nadadeira dorsal terminando em ponta e anal arredondada. Caudal ovalada e contorno da peitoral arredondado. Limite posterior da peitoral sobre a vertical que passa pela base da pélvica, e esta quando dobrada para trás alcançando a papila uro-genital. Origem da dorsal na vertical do 4 ?, 5 o ou 6 ? raio da anal.

Dados merísticos são apresentados na tab. 1 (com exceção das escamas em torno do pedúnculo que são $12 \mathrm{em}$ todos os exemplares) e dados morfométricos na tab. 3.

Comportamento reprodutivo: como o descrito para $C$. minimus.

Forma do ovo: como o de C. minimus.

Notas ecologicas: Encontrada em ambiente semelhante aos descritos para C. minimus, em simpatria com Callichthys callichthys L., 1758 e Cynolebias whitei Myers, 1942.

Localidade-tipo: Brejo próximo a Inoã, Município de Maricá, RJ. 
Vol. 5(4), 1988

Distribuição: conhecida apenas na localidade-tipo (fig. 5).

Etimologia: Do Latim fractus (fratura) + fasciatus (com faixas), referindose ao padrão de faixas da nadadeira caudal dos machos.

Material: Holótipo ơMZUSP 36423. Parátipos - MZUSP 36424 (6 o, 5 \&); MNRJ 11292 ( 1 o*, 1 \%); CAGC 123 (5 o*, 14 \%).

Observação: uma referência de coleta e uma fotografia desta espécie aparecem em La Corte (1982).

\section{Cynolebias cruzi sp.n.}

Figs. 3 e $4 g-i$

Diagnose: espécie que se distingue facilmente das demais espécies do complexo $C$. minimus pelo menor comprimento da base e número modal de raios das nadadeiras dorsal e anal (média da base da dorsal com 19,1\% do comprimento padrão em machos e $16,9 \%$ em fêmeas, e moda de 13 raios em ambos sexos; média da base da anal com $24,6 \%$ do comprimento padrão em machos e $19,3 \%$ em fêmeas, e moda de 15 raios em ambos sexos), coloração geral do corpo escura e faixas das nadadeiras pouco conspícuas.

Descrição dos machos: exemplares examinados entre 13,5 e $22,9 \mathrm{~mm}$ de comprimento padrão.

Flanco de coloração verde-bronzeada e região opercular e íris, verde-limãometálica, com cerca de 14 faixas verticais no tronco, três da região opercular, uma atravessando o olho e contorno das escamas de coloração vermelho-pardoescura. Desta cor, estão presentes cerca de 5 faixas na porção anterior da nadadeira caudal, nove na anal e nove na dorsal, sobre fundo castanho claro, havendo pouco contraste de cores ou até mesmo sendo as faixas imperceptíveis em alguns exemplares. Pélvica castanha e peitoral transparente.

Nadadeiras dorsal e anal terminando em ponta, caudal algo truncada (sempre menor do que a de $C$. minimus e $C$. fractifasciatus, mas de difícil medição em exemplares fixados) (Fig. 4) e peitoral de contorno arredondado. Limite posterior da peitoral sobre a vertical que passa pela base da pélvica e esta quando dobrada para trás alcançando a base da anal. Origem da dorsal na vertical do 49,5 . ou 60 raio da anal. Peitoral com papilas pouco conspícuas na parte anterior da face interna dos raios superiores.

Dados merísticos são apresentados na tab. 1 (com exceção das escamas em torno do pedúnculo que são 12 em todos os examplares) e dados morfométricos na tab. 4.

Descrição das fêmeas: exemplares examinados entre 15,4 e $20,8 \mathrm{~mm}$ de comprimento padrão.

Coloração do corpo castanho-claro-uniforme (mais escura do que em fêmeas de $C$. minimus e $C$. fractifasciatus), com nadadeiras transparentes. Região opercular e porção anterior ventral rosadas e com reflex os verde-claros. Íris amarela e uma faixa vertical castanho-clara atravessando o olho. Nadadeira dorsal terminando em ponta, anal arredondada, caudal ovalada e contorno da peitoral arredondado. Limite posterior da peitoral sobre uma vertical que passa pela base da pélvica, e esta quando dobrada para trás, alcançando a papila uro-genital. Origem da dorsal na vertical do 39,40 ou 50 raio da anal. 
Revta bras. Zool.

Dados merísticos são apresentados na tab. 1 (com exceção das escamas em torno do pedúnculo que são $12 \mathrm{em}$ todos os examplares) e dados morfométricos na tab. 4.

Comportamento reprodutivo: como o descrito para $C$. minimus.

Forma do ovo: único ovo examinado semelhante ao de $C$. minimus, distinguindo-se por apresentar prolongamentos do córion proporcionalmente maiores (comprimento igual a $28,8 \%$ do raio do ovo).

Notas ecologicas: encontradas em ambiente semelhante ao descrito para C. minimus, a aproximadamente $100 \mathrm{~m}$ de distância do mar, junto à vegetação de restinga. Callichthys callichthys L. 1758, Cynolebias whitei Myers, 1942 e Cynolebias constanciae Myers, 1942, vivem em simpatria com Cynolebias cruzi.

Localidade-tipo: brejo próximo a Barra de São João, Município de Casimiro de Abreu, RJ.

Distribuição: conhecida apenas na localidade-tipo (Fig. 5).

Etimologia: nome em homenagem ao Prof. Carlos Alberto G. Cruz, que nos últimos anos tem se dedicado ao estudo do gênero Cynolebias.
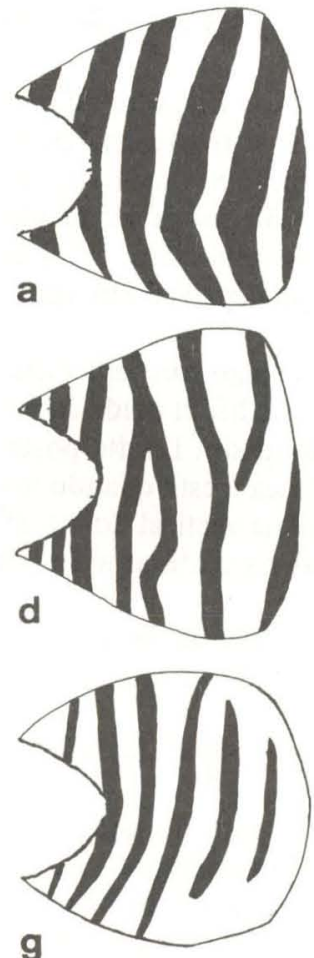
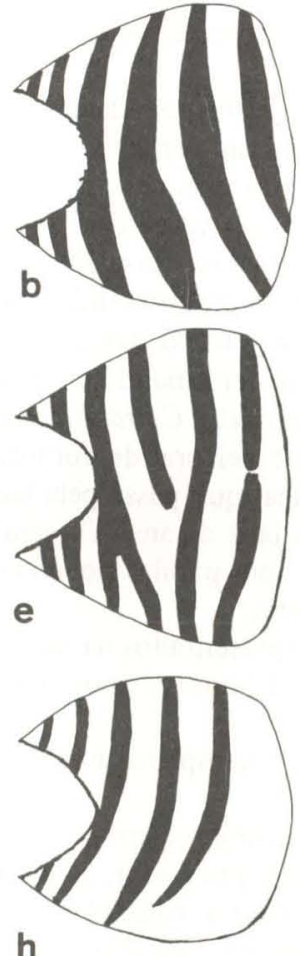
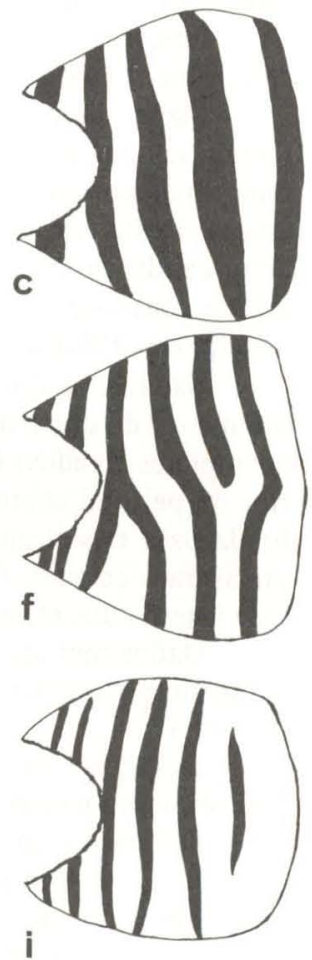

FIG. 4 - Padrões de faixas de nadadeiras caudais em machos do complexo $C$. minimus: a - c - Cynolebias minimus Myers, 1942; d - $\mathrm{f}$-Cynolebias fractifasciatus sp. $\mathrm{n}$.; $\mathrm{g}-\mathrm{i}-$ Cynolebias cruzi $\mathrm{sp} . \mathrm{n}$. 
Vol. 5(4), 1988

Material: Holotipo ơ MZUSP 36297. Parátipos: MZUSP 36298 (5 ઠ, 3 q),

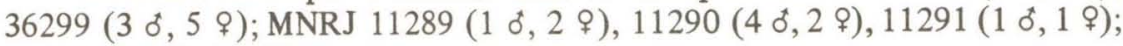
CAGC $124(9$ ơ, 5 \%).

Observação: uma referência de coleta e uma fotografia desta espécie aparecem em La Corte $(1980 ; 1982)$.

TAB. 1 - Dados merísticos das três espécies do complexo C. minimus.

\begin{tabular}{|c|c|c|c|c|c|c|c|c|c|c|c|c|c|c|c|c|c|c|}
\hline & \multicolumn{6}{|c|}{$\begin{array}{l}\text { raios nad. dors. } \\
111213141516\end{array}$} & \multicolumn{7}{|c|}{ raios nad. anal } & \multicolumn{5}{|c|}{$\begin{array}{l}\text { esc. lin. long. } \\
2223242526\end{array}$} \\
\hline$\underset{\delta}{\operatorname{minimus}}$ & & 2 & 41 & & 9 & 1 & & & 5 & 71 & 0 & 6 & & & & 14 & 5 & 1 \\
\hline$\underset{\uparrow}{\operatorname{minimus}}$ & & & 3 & 71 & & 3 & & & 2 & 6 & 9 & 3 & 2 & & 3 & 12 & 5 & 1 \\
\hline $\begin{array}{c}\text { fractif. } \\
\delta\end{array}$ & & & 2 & 5 & 2 & 2 & & & 2 & 2 & 4 & 3 & 2 & 2 & 1 & 5 & 1 & 1 \\
\hline $\begin{array}{c}\text { fractif. } \\
\uparrow\end{array}$ & & 1 & 1 & 7 & 9 & 2 & & & & 1 & 51 & & 3 & & 3 & & 2 & \\
\hline $\begin{array}{c}\text { cruzi } \\
\delta\end{array}$ & 1 & 21 & & 6 & & & & 13 & 5 & 3 & & & & 1 & & 10 & 4 & \\
\hline $\begin{array}{c}\text { cruzi } \\
\uparrow\end{array}$ & & 7 & 8 & 4 & & & 3 & 9 & 4 & 2 & & & & 1 & 3 & 9 & 3 & 2 \\
\hline
\end{tabular}

TAB. 2 - Dados morfométricos de Cynolebias minimus Myers, 1942 ( $\overline{\mathrm{X}}=$ média; $\mathrm{s}=$ desvio padrão da mêdia; $\mathrm{n}=$ número de indivíduos medidos).

\begin{tabular}{lcccccccc} 
& \multicolumn{3}{c}{ machos } & \multicolumn{5}{c}{ fêmeas } \\
& variação & $\overline{\mathbf{X}}$ & $\mathbf{s}$ & $\mathbf{n}$ & variação & $\overline{\mathbf{X}}$ & $\mathbf{s}$ & $\mathbf{n}$ \\
alt. corpo & $23,1-30,5$ & 26,6 & 1,6 & 28 & $21,4-28,2$ & 25,7 & 1,9 & 22 \\
comp. cab. & $26,5-30,7$ & 28,7 & 1,1 & 26 & $26,6-31,3$ & 28,7 & 1,3 & 23 \\
larg. cab. & $18,9-23,4$ & 20,5 & 1,3 & 28 & $18,8-23,6$ & 21,0 & 1,4 & 23 \\
alt. cab. & $19,4-25,8$ & 22,3 & 1,6 & 28 & $18,1-24,3$ & 21,2 & 1,5 & 23 \\
diam. olho & $31,0-42,1$ & 36,2 & 2,3 & 27 & $33,3-42,6$ & 37,7 & 2,4 & 23 \\
alt. ped. & $11,8-17,2$ & 14,4 & 1,3 & 28 & $11,0-13,9$ & 12,6 & 0,9 & 23 \\
comp. p.-dor. & $55,7-64,5$ & 59,8 & 2,0 & 28 & $57,4-54,8$ & 61,9 & 1,5 & 23 \\
comp. p.-pel. & $44,4-49,2$ & 47,2 & 1,4 & 27 & $47,5-54,0$ & 51,0 & 1,9 & 23 \\
c. bas. n. dor. & $20,7-27,6$ & 23,7 & 1,8 & 27 & $18,2-25,2$ & 22,8 & 2,0 & 23 \\
c. bas. n. an. & $26,1-34,5$ & 29,6 & 1,9 & 28 & $21,2-25,8$ & 23,2 & 1,5 & 23
\end{tabular}


Revta bras. Zool.

TAB. 3 - Dados morfométricos de Cynolebias fractifasciatus sp.n. $\overline{\mathbf{X}}=$ média; s $=$ desvio padrão da média; $\mathrm{n}=$ número de indivíduos medidos).

\begin{tabular}{lcccccccc} 
& \multicolumn{3}{c}{ machos } & \multicolumn{5}{c}{ fêmeas } \\
& variação & $\overline{\mathbf{X}}$ & s & n & variação & $\overline{\mathbf{X}}$ & s & n \\
alt. corpo & $22,1-28,5$ & 25,9 & 1,8 & 13 & $23,1-27,1$ & 24,2 & 1,1 & 20 \\
comp. cab. & $27,6-31,7$ & 29,9 & 1,0 & 13 & $27,2-32,8$ & 30,2 & 1,8 & 20 \\
larg. cab. & $19,2-21,6$ & 20,6 & 0,7 & 1.3 & $18,1-22,6$ & 20,5 & 1,2 & 20 \\
alt. cab. & $20,9-26,1$ & 23,5 & 1,6 & 13 & $19,8-23,2$ & 21,5 & 1,0 & 20 \\
diam. olho & $33,6-45,2$ & 38,7 & 3,3 & 13 & $34,0-43,2$ & 38,2 & 2,4 & 20 \\
alt. ped. & $11,6-14,9$ & 13,5 & 1,0 & 13 & $9,9-13,6$ & 12,3 & 1,0 & 20 \\
comp. p.dor. & $59,0-54,3$ & 60,6 & 1,5 & 13 & $59,3-69,0$ & 64,2 & 2,4 & 20 \\
comp. p.pel. & $43,2-48,9$ & 46,6 & 1,9 & 12 & $48,5-54,5$ & 50,9 & 1,6 & 19 \\
c. bas. n. dor. & $19,0-25,7$ & 22,2 & 2,0 & 13 & $16,9-20,9$ & 19,2 & 1,2 & 20 \\
c. bas. n. an. & $25,7-34,5$ & 29,9 & 2,7 & 13 & $20,5-25,8$ & 23,0 & 1,7 & 20
\end{tabular}

TAB. 4 - Dados morfométricos de Cynolebias cruzi sp. $\mathrm{n}(\overline{\mathrm{X}}=$ média; $\mathrm{s}=$ desvio padrão da média; $\mathrm{n}=$ número de indivíduos medidos).

$\begin{array}{lllllllll}\text { alt. corpo } & 23,8-30,4 & 26,4 & 1,8 & 20 & 23,0-28,7 & 25,5 & 1,7 & 17 \\ \text { comp. cab. } & 28,7-32,7 & 30,3 & 1,1 & 20 & 27,7-31,3 & 29,3 & 1,2 & 18 \\ \text { iarg cab. } & 18,8-22,5 & 20,2 & 1,1 & 21 & 18,8-23,2 & 20,7 & 1,3 & 18 \\ \text { alt. cab. } & 20,0-24,6 & 21,8 & 1,2 & 21 & 18,2-22,8 & 20,9 & 1,3 & 18 \\ \text { dian. olho } & 33,0-42,2 & 36,3 & 3,0 & 20 & 31,1-44,4 & 38,8 & 3,4 & 18 \\ \text { alt.ped. } & 11,5-14,7 & 13,3 & 0,8 & 21 & 10,3-12,5 & 11,7 & 0,7 & 18 \\ \text { comp.p.-dor. } & 58,2-65,3 & 60,9 & 1,6 & 21 & 60,4-66,0 & 63,7 & 1,8 & 18 \\ \text { comp.p.-pel. } & 46,4-54,4 & 48,8 & 1,9 & 21 & 47,4-54,6 & 51,8 & 2,2 & 17 \\ \text { c.bas.n.dor. } & 16,4-21,6 & 19,1 & 1,2 & 20 & 14,9-20,1 & 19,3 & 0,8 & 18 \\ \text { c.bas.n.an. } & 22,3-27,5 & 24,6 & 1,3 & 21 & 17,9-21,4 & 19,3 & 3,0 & 18\end{array}$

\section{DISCUSSÃO}

O complexo de espécies Cynolebias minimus é aqui hipotetizado constituir um grupo monofilético (sensu Hennig, 1966), devido aos machos compartilharem a presença de nadadeira caudal ligeiramente truncada. As demais espécies do grupo Leptolebias, assim como outras do gênero Cynolebias que exibem caracteres morfologicos aparentemente plesiomórficos e mais semelhantes a Leptolebias, os machos possuem nadadeira caudal redonda, ovalada ou lanceolada. A forma dos prolongamentos do córion descritas para o complexo de espécies é completamente distinta dos das outras espécies de Cynolebias ou outro rivulídeo já observados (em forma de espinho). Dentre estes, so foi possível até o momen- 
Vol. 5(4), 1988

to examinar ovos de duas espécies de Leptolebias (C. sandrii (Faria \& Muller, 1937) e $C$. aureoguttatus Cruz, 1974), que igualmente não possuem prolongamento em forma de cogumelo. Tal forma por tanto deve ser uma sinapomorfia do complexo $C$. minimus. Outros caracteres descritos compartilhados pelo complexo, são de ocorrência bastante generalizada no gênero, sendo considerados simplesiomorfias.

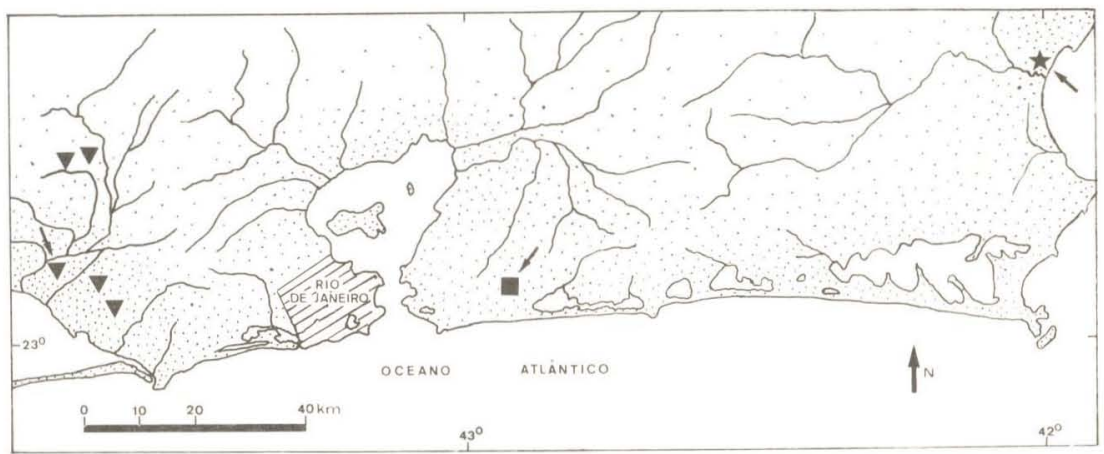

FIG. 5 - Distribuição de espécies do complexo Cynolebias minimus. Triângulos: C. minimus Myers, 1942; quadrado: $C$. fractifasciatus sp. n.; estrela: $C$. cruzi sp. n.; setas: localidades-tipo.

Os muitos caracteres merísticos e morfométricos compartilhados entre $C$. minimus e $C$. fractifasciatus, quando comparados com as restantes espécies de Leptolebias (C. marmoratus (Ladiges, 1934), C. sandrii (Faria \& Muller, 1937), C. aureoguttatus Cruz, 1974 e $C$. nanus Cruz \& Peixoto, 1984) são de ocorrência generalizada, sendo considerados primitivos. Entretanto, os machos de $C$. fractifasciatus e $C$. cruzi apresentam média da altura do pedúnculo caudal $(13,5$ e 13,3 respectivamente) abaixo da do macho de $C$. minimus $(14,4)$ e de outras Leptolebias spp. O limite posterior das nadadeiras peitorais dos machos se situam numa vertical sobre a base das pélvicas em $C$. cruzi e entre estas e o ânus em $C$. fractifasciatus, enquanto em $C$. minimus e todas outras Leptolebias spp, o limite das peitorais dos machos atinge a base da papila uro-genital. Assim, é levantada a hipótese de $C$. fractifasciatus e $C$. cruzi serem mais relacionadas entre si do que com $C$. minimus, como ilustra o cladograma da fig. 6.

São considerados caracteres antapomórficos os padrões de colorido da nadadeira caudal de $C$. minimus e o de $C$. fractifasciatus e a redução no comprimento da base e número modal de raios da nadadeira anal de $C$. cruzi.

Outras espécies não descritas poderão acrescentar informações significativas para a compreensão filogenética e biogeográfica do grupo.

Huber (1981), menciona o encontro de exemplares de Cynolebias em Magé e entre Rio Bonito e Tanguá, localidades no Rio de Janeiro, que pela ligeira descrição parecem pertencer ao complexo $C$. minimus. 
Revta bras. Zool.

Os padrões de colorido da cauda dos machos de $C$. minimus ilustrados aqui, são semelhantes ao desenhado por Constance White (desenho original não publicado; cópia em preto e branco em La Corte, 1982), de um exemplar coletado na localidade-tipo, no qual Myers se baseou na descrição, e aos das fotos da descrição original de C. ladigesi Foersch, 1958.

Porém, o padrão exibido por exemplares identificados como Gynolebias NSC2 que aparecem em fotografias de artigos recentes (e.q., Remeur, 1982a), é semelhante ao de $C$. fractifasciatus. Neste caso, devido à falta de informações sobre a origem dos exemplares, fica-se em dúvida se são resultados de seleções, ou hibridizações em cativeiro, ou se até mesmo pertencem a uma espécie distinta.

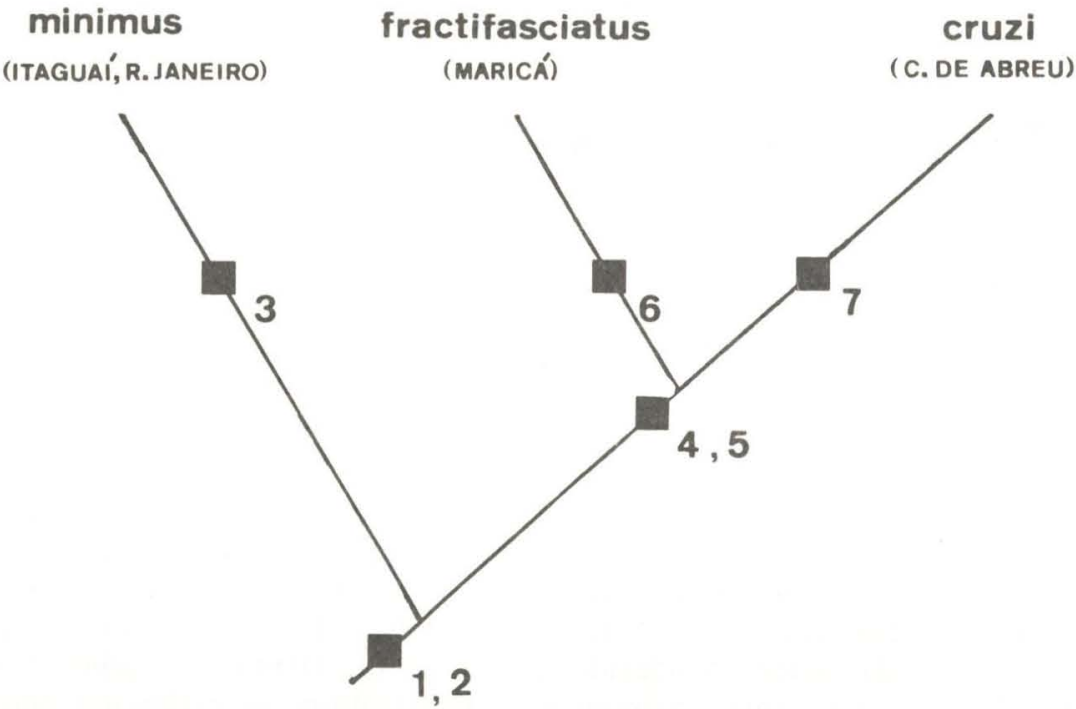

FIG. 6 - Cladograma de relações filogenéticas das espécies do complexo Cynolebias minimus e indicações das áreas de ocorrência. Caracteres derivados apresentados: 1 nadadeira ligeiramente truncada nos machos; 2 - córion com prolongamento em forma de cogumelo; 3 - padrão de colorido da nadadeira caudal dos machos; 4 - redução da média da altura do pedúnculo caudal; 5 - redução do comprimento da nadadeira peitoral dos machos; 6 - padrão de faixas da nadadeira caudal dos machos; 7 - redução do comprimento da base e de número de raios da nadadeira anal.

\section{AGRADECIMENTOS}

Aos Dr. Naércio A. Menezes e Heraldo A. Britsky, meus especiais agradecimentos pela leitura do manuscrito e oportunas sugestões. Sou grato ao Dr. Ubirajara R. Martins, pela revisão de parte do manuscrito; ao Prof. Carlos A.G. Cruz, pela sua gentileza em deixar à minha disposição a sua coleção; aos Profs. Gustavo W. Nunan e Priscila A. Grohmann, pela hospitalidade em me acolher em seus la- 
Vol. 5(4), 1988

boratorios; ao apoio do Prof. Sérgio R.P. Annibal; aos Srs. Marco T. C. Lacerda, Mário C. C. Pinna, Tereza C. R. Silva e Kenny Tanizaki Fonseca, pela ajuda em campo; e, ao Sr. João O. Alves, pelas fotografias.

\section{REFERENCIAS}

Carvalho, A.L. 1957. Notas para o conhecimento da biologia dos peixes anuais. Revta bras, Biol., 17(4):459-466.

Cruz, C.A.G. 1974. Sobre Cynolebias aureoguttatus Myers, 1952 (Osteichthyes, Cyprinodontidae, Rivulinae). Arq. Univ. Fed. Rur. Rio de Janeiro, 2(4):19-21.

Cruz, C.A.G. 1983. Uma nova espécie de Cynolebias do Estado do Espírito Santo, Brasil (Pisces, Cyprinodontidae). Papeis Dep.Zool. S. Paulo, 35(6):73-77.

Cruz, C.A.G. \& O. Peixoto. 1983. Novo Peixe Anual do Estado do Rio de Janeiro, Brasil (Pisces, Cyprinodontidae). Arq. Univ. Fed. Rur. Rio de Janeiro, 6(1):89-93.

Foersch, W. 1958. Beobachtungen and Erfahrungen bei der Pflege und Zucht von Cynolebias ladigesi Myers. Aquar. Terr. Zeit., 11(9):257-260.

Fowler, H.W. 1954. Os peixes de água doce do Brasil (40 entrega). Arq. Zool. Est. São Paulo 9:1-IX + I-400.

Hennig, W. 1966. Phylogenetic systematics. Urbana, Univ. Illinois Press, 263 pp.

Huber, H.H. 1981. Cynolebias heloplites n. sp. Killi Revue 8 (5) (Suppl.): 1-15.

La Corte, R.S. 1980. Introducing Cynolebias constancieae. Freshwater Mar. Aquar., 3 (7): $10-12,86,88-89$.

La Corte. 1982. Natural history notes on some Cynolebias species. J. Amer. Killifish Assoc., 15(5):215-227.

Ladiges, W. 1955. Neue Fische aus Zentral-Brasilien. Aquar. Terr. Zeit. 8(9):230-232.

Lazara, K.J. 1982. The taxonomy and nomenclature of South and Central American Killifishes. J. Amer. Killifisch Assoc., 15(5):166-184.

Lazara, K.J. 1984. Killifish Master Index. Third Edition. American Killifish Assoc., Cincinnati, Ohio, 295 pp.

Myers, G.S. 1942. Studies on South American fresh-water fishes. Stanford Ichthyol. Bull., 2(4):89-114.

Myers. G.S. 1952. Annual fishes. Aquar. J., 23(7):125-141.

Parenti, L.R. 1981. A phylogenetic and biogeographic analysis of cyprinodontiform fishes (Teleostei, A therinomorpha). Bull. Amer. Mus. Nat. Hist., 168(4):335-557.

Remeur, A. 1981a. Le genre Cynolebias, Steindachner 1876. Aquarama, 58:32-36, 74.

Remeur, A. 1981b. Le genre Cynolebias, Steindachner 1876. Aquarama, 59:26-29, 70-72

Remeur, A. 1981c. Le genre Cynolebias, Steindachner 1876. Aquarama, 62:24-25, 72. 
Revta bras. Zool.

Remeur, A. 1982a. Le genre Cynolebias, Steindachner 1876. Aquarama, 63:22-25.

Remeur, A. 1982b. Le genre Cynolebias, Steindachner 1876. Aquarama, 64:20-23, 47.

Schroeter, L. 1956. Cynolebias (Cynopoecilus) splendens Myers - ein neuer farbenprächtiger Aquarienfisch aus Brasilien. Aquar. Terr. Zeit., 29(12):63-66.

Seegers, L. 1980. Killifische, eierlegende Zahnkarpfen im Aquarium. Verlag Eugen Ulmer, Stuttgart, $174 \mathrm{pp}$.

Siegel, G. 1958. Interessante Neuigkeiten über bodenlaichende südamerikanische Zanhkarpien. Aquar. Terr. Zeit., 11(9):257-260.

Vaz-Ferreira, R. \& B. Sierra. 1973. El genero Cynolebias Steindachner, 1876 (Atheriniformes, Cyprinodontidae): caracteres, especies y distribucion. Trab.V. Congr. Latinoamer. Zool., 1:245-260.

Vaz-Ferreira, R. \& B. Sierra. 1974. Campellolebias brucei n. gen. n. sp., cyprinodontido con especializacion de la papila genital y de los primeros radios de la aleta anal. Com. Zool. Mus. Hist. Nat. Montevideo, 10(138):1-16.

Vaz-Ferreira; B. Sierra \& S. Scaglia-de-Paulete, 1964. Tres especies nuevas del genero Cynolebias Steindachner, 1876 (Teleostomi, Cyprinodontidae). Com.Zool. Mus. Hist. Nat. Montevideo, 8(102):1-48.

Weitzman, S.H. 1962. How Cynolebias ladigesi got its name. Am.Kj llifish Assoc., Killie Notes, 1(2):12-13.

Weitzman, S.H. \& J.P. Worms. 1967. Sou th American Cyprinodont Fishes Allie to Cynolebias with a Description of a New Species of Austrofundulus from Venezuela. Copeia, 1967(1):89-100. 